\title{
Developing a Fuzzy Multi-attribute Matching and Negotiation Mechanism for Sealed-bid Online Reverse Auctions
}

\section{Kune-muh Tsai ${ }^{1}$ and Feng-chin $\mathrm{Chou}^{2}$}

${ }^{1}$ National Kaohsiung First University of Science and Technology, Department of Logistics Management, kmtsai@ccms.nkfust.edu.tw

${ }^{2}$ National Kaohsiung First University of Science and Technology, Institute of Management

${ }^{2}$ WuFeng University, Department of Hospitality Management, fcchou@wfu.edu.tw

Received 10 November 2010; received in revised form 7 March 2011; accepted 26 April 2011

\begin{abstract}
Online reverse auctions have benefits in reducing business purchasing costs and lead times. Previous research focused on developing new mechanisms for open-cry and multiple rounds of auctions, which is not appropriate for direct goods purchasing. Moreover, the implementation issues in the web-site design are not considered. We construct an easily-computed fuzzy multi-attribute matching mechanism for sealed-bid and single round auctions, which include price and attributes like quality, due dates, credit rating, etc, and are suitable for procuring direct input goods. To improve the negotiation quality, an aftermath negotiation mechanism is also developed so that the auctioneer can negotiate with the bidders for certain attribute values when the bid does not meet the requirement of the auctioneer. We further build an online fuzzy negotiation system (FNS) to evaluate the proposed mechanism.
\end{abstract}

Keywords: Online reverse auctions, Multi-attribute, Sealed-bid, Fuzzy sets, Negotiations 


\section{Introduction}

The growth of Internet technologies conduces to wide spread use of online reverse auctions for B2B (Business-tobusiness) transactions. An e-marketplace in B2B is composed of three main parties: suppliers, buyers, and market intermediaries. The suppliers desire to attain more profits/revenues by approaching more customers, while the buyers attempt to find an economic way to place orders [23]. In many companies, online reverse auctions have yielded savings in terms of price reductions as well as improvements in the purchasing process [3], [16]. Prior research indicated that online reverse auctions for standardized products can have benefits in purchase price savings for buyers ranging from $5 \%$ to $20 \%$ [1], [23]. Since online reverse auctions can lead to reduced order cycle time and increased productivity [4], it has obtained considerable attention [9], [23], [31]. Furthermore, the success of online reverse auctions can help buyers diversify their supplier base to hedge against risks, and also help suppliers reduce operational and customer acquisition costs [12]

Transactions between enterprises are complicated. The seller and buyer need to evaluate and negotiate with each other and consider many factors during transactions. There are several types of auction protocols; two of the most popular forms are the ascending price English auction where the lowest price of bid wins and the descending price Dutch auction where the highest price of bid wins [9]-[10], [33]. Some other types of classification includes: single or multiple units of auctions; single or multiple attributes of auctions; open-cry or sealed-bid of auctions; single or multiple rounds of auctions, etc. In the open-cry auction, full price visibility is made available to all participants; while in the sealed-bid, bidders have no visibility of the price submitted [23], [32]. Traditional reverse auctions are performed in a sealed-bid and single unit form, which is still common nowadays for sourcing of direct input goods or engineering projects [32]-[33]. In procuring direct input goods, companies usually implement with a single round and sealed-bid tendering protocol since those suppliers are different from general goods suppliers in that the former require special certification process to make sure the quality, credit, delivery etc. meet the requirement of the buyer [2], [5]-[6], [10]. The auctioneer and bidders for this kind of goods cooperate rather than compete with each other to pursue for the long-term relationship and procedural fairness [25]; the tendered bids usually can be matched (but not negotiated) in a single round. For indirect goods, like MRO (maintenance, repair and operations) goods, procurement can be performed with an open-cry and multiple rounds of auction in order to achieve as low as possible the price, which has attracted a lot of attention recently [2], [15], [26], [32]-[33]. The auctioneer and bidders for indirect goods tend to be self-interest oriented since their primary concern is the respective interests instead of the relationship [13][14]; the negotiation process (but not matching process) usually proceeds with several rounds. Moreover, multiple attributes are considered in business procurement [13]-[14], [32]-[33], such as the unit price of the product, delivery time, quality, warranty, etc., and different issues are accorded different weights by negotiators. It enables trade-offs between different attributes, such that the two parties can arrive at a mutually beneficial agreement. This study aims at developing a reverse auction mechanism for direct input goods in a sealed-bid and single round environment.

\section{Background and Focuses}

There were some studies conducted in reverse auctions with multi-attribute negotiations. Teich et al. [32]-[33] studied an e-auction mechanism in a multi-round and open-bid environment; they considered not only price and quantity combinations, but also other relevant attributes. However, they only provide a prototype instead of explicit mechanism. Leskela et al. [26] later extended the work of Teich et al. [33] to multi-unit combinatorial auctions and Ervasti et al. [11] further improved that of Leskela et al. [26] with the Group Support Mechanism for the efficiency of combinatorial auctions. In Bichler and Kalagnanam [2], they implemented with mixed-integer programming (MIP) formulation to study configurable multi-attribute auctions for single or multiple sourcing with order allocations mechanism to determine winning bidders. The mechanism is applied in a multi-round and open-cry environment. However, the MIP formulation makes the auction mechanism difficult to solve. To study the sealed-bid, multiple-issue reverse auction problem, Cheng [6] presented a multi-attribute decision making problem for buyers to choose among the bidders with the TOPSIS approach proposed by Hwang and Yoon [20]. He also implemented a multiple-objective model for bidders and included the available-to-promise (ATP) inventory in the model. Despite this many practical problems were considered, the computation procedure of his models is not straightforward, which limits its implementation to online auction systems.

Multi-agents are widely used in modeling auction protocols (e.g., [7], [10], [13]-[14], [27], [29]-[30], [32], [34], [35]); however, except some exceptions (e.g., [34], [35]), most are conducted with protocol simulation to test how well their negotiation mechanisms are as compared to others (e.g., [7], [10], [14], [27], [29]-[30]). Even though some of the negotiation protocols of those studies can be referenced for reverse auctions, the development environments and requirements are completely different from those of the online reverse auctions. In addition, many of those protocols are more applicable to one-to-one negotiation rather than to one-to-many or many-to-one's. Among those studies, Jennings and their research team (e.g., [13], [14], [29]) performed a series of significant research in multi-issue negotiation with self-interested software agents to examine the negotiation protocols as well as the negotiation behaviors from game theory perspectives. Teuteberg [34] implemented a multilateral negotiation model with fuzzy preferences on an agent-based job-marketplace. Nassiri-Mofakham [27] considered adding personality into multiagent models that agents can act more closely to human-like conducts to enhance the realism of rational bargaining 
behaviors. Wang et al. [36] presented with autonomous agents a 2-phase evaluation model, a parallel dispatch model and an auction-like negotiation model for B2C e-marketplaces. David, et al. [10] proposed a sealed-bid, multiattribute reverse auction model and considered three protocols, but they did not include detailed implementation issues. Shih et al. [30] proposed an automated negotiation model using a mobile reverse auction agent system to mediate between the buyer and sellers and to execute bidding asynchronously, autonomously and repetitively. Cheng et al. [7] proposed an agent-based heuristic model for making trade-offs in automated negotiations by fuzzy inference systems

The above works mainly studied auction mechanisms that are appropriate for general goods, like MRO, and are not suitable for direct input goods, which accounts for major purchasing amounts in industries [5]-[6], [8], [10], [20]. Our study focuses on developing a matching mechanism for reverse auctions of direct input goods or engineering projects in a B2B business environment. The mechanism is to be applied in a single round, single unit and sealedbid auction environment with multi-attribute, which is the first focus of our study.

While the product attributes negotiated between the buyer and seller in an e-marketplace can be expressed in crisp values, this kind of expression is not proper to describe the requirement clearly in practice [34] because human judgments and preferences are often vague and cannot be estimated with exact numerical value. As claimed by Gu and Zhu [18], a more realistic approach may be to use linguistic assessments instead of numerical values. Most current B2B reverse auction sites implement with straightforward arithmetic operations like add, subtract and multiplication, etc. to choose among the submitted bidders [32] and ignore human uncertainty in making judgments and expressing their preferences, which might deviate the real intention of bidders and auctioneers. In the literature, the application of fuzzy sets theory in real world decision-making problems has given very good results because it provide a more flexible solution framework [7], [18], [34], [36]. Thus, the second focus of our study is to implement the fuzzy sets theory to model the multiple attributes to reflect the linguistic representation of buyers and sellers in auctions.

Foroughi et al. [15] mentioned that as a supplier faces the most important customers, they will bid more aggressively and may be willing to settle for a lower price than when facing with less important customers. As a result, in the transaction process of a buyer and a supplier, both parties may adopt different negotiation attributes and weights according to both relationship-based and market-based coordination [17]. Kumar et al. [25] stated that enhanced procedural fairness can maintain relationship quality and is the basis of sustained competitive advantage. Daly and Nath [8]-[9] cited previous research to support post-auction negotiations that are beneficial to maintain procedural fairness. Therefore, the post-auction negotiations can be planned as an additional step before final supplier selection [8]-[9], [35]. Not only has our study developed a multi-attribute negotiation mechanism, we also have incorporated an aftermath negotiation mechanism in reverse auctions to implement the integrative negotiation strategy with a hope to fulfill a 'win-win' goal. Therefore, the third focus of our study is to augment an aftermath negotiation mechanism in addition to the fuzzy multi-attribute matching mechanism such that the buyer and sellers can negotiate after the auction has ended.

The preceding studies (e.g. [6]-[7], [10], [13]-[14], [27], [29], [32]-[33]) about the negotiation mechanisms of online reverse auctions mainly adopted negotiation agents or mathematical optimization models for auctions like MRO or eBay type of products, which requires several rounds of negotiation to reach equilibrium for both parties. Those negotiation mechanisms are not appropriate for our focus in direct input goods. Furthermore, those mechanisms are mainly developed either with mathematical programming models (e.g. [6], [10], [32]-[33]) or with multi-agent protocols (e.g. [7], [13]-[14], [27], [29]); hence, they cannot be easily embedded in the web-site design. Actually, if an aftermath negotiation mechanism were to be added up to their studies, it would complicate the computation procedures. In contrast, our fuzzy auction mechanism can be handily integrated into an existing reverse auction website for direct input goods or outsourcing of projects. As the strategic supplier fails to render the best bid, the buyer can alternatively negotiate with them to obtain an appropriate transaction with our aftermath negotiation mechanism to create a win-win situation.

\section{Fuzzy Matching and Negotiation Mechanism Development}

This study focuses on developing a multi-attribute single-round bidding mechanism with aftermath negotiations. In a reverse auction for direct input goods or engineering projects via Internet, a tendered bid is usually initiated by a buyer or an auctioneer who specifies the requirements with attributes like quantity, quality, due dates, prices, etc. The bid information is then broadcasted via Internet to potential suppliers who are bidders in the reverse auction. Those potential suppliers are usually invited, and before participating in the reverse auction, they are verified by the buyer. Qualified bidders read the bid information and those who are interested propose their bid offers to the reverse auction systems. The systems then match the tendered bid requirements of the buyer with bid offers through the matching mechanism.

The bidding process of our study is divided into three stages, as Figure 1 shows. In the first stage, a request is proposed by a buyer, and different weights are given according to the importance levels of attributes the buyer emphasizes. In the second stage, several sellers provide their bid offers based on the buyer's request. In the third stage, the fuzzy negotiation matching mechanism selects the optimal bid as a reference for decision makers, based 
on the buyer's requests and bid offers from sellers. Further, our system considers the situation when the buyer is dissatisfied with the offer of the winning bid (i.e., lower than the lowest threshold value of the buyer). When this happens, the system initiates the aftermath negotiations for the buyer to negotiate the transaction terms agreed upon by both parties. In order to express the non-quantifiable meaning and perception in negotiation attributes, the reverse auction systems utilize fuzzy sets theory to define the negotiation terms of attributes as well as the matching mechanism.

Assume that $A_{i}=\operatorname{tri}\left(a_{i}, b_{i}, c\right)$ is a fuzzy triangular number, where $i \in[1, \cdots \cdots, n]$ and $\left(a_{i}, b_{i}, c_{i}\right)$ denotes the values of the three apexes of the triangle from left to right. According to the characteristics of fuzzy triangular numbers and the extension principle, the addition and subtraction of any two fuzzy triangular numbers of $A_{1}$ and $A_{2}$, and the intersection of a constant $\lambda$ and a fuzzy triangular number are as follows [24].

$$
\begin{aligned}
& A_{1}+A_{2}=\operatorname{tri}\left(a_{1}+a_{2}, b_{1}+b_{2}, c_{1}+c_{2}\right) \\
& A_{1}-A_{2}=\operatorname{tri}\left(a_{1}-a_{2}, b_{1}-b_{2}, c_{1}-c_{2}\right) \\
& \lambda \wedge A_{1}=\operatorname{tri}\left(\lambda a_{1}, \lambda b_{1}, \lambda c_{1}\right)
\end{aligned}
$$

Defuzzification process converts fuzzy numbers into representative crisp values. Literature reveals many defuzzification methods, such as the centroid of area, bisector of area, mean of maximum, etc. [21]. The centroid of a triangle is the average value of the three apexes of the triangle [24]. This study employs the centroid of area as the defuzzification method to reduce the complexity of computation. If other fuzzy functions are implemented, we only need to compute the centroids of them to obtain the crisp values as the defuzzification values. For $A_{i}$ in Eq.(1), the defuzzification of the triangular-shape fuzzy number $d f\left(A_{i}\right)$ is as follows:

$$
d f\left(A_{i}\right)=\left(a_{i}+b_{i}+c_{i}\right) / 3
$$

This study employs the triangular-shape fuzzy number for ease of computation and closeness of the computed results than if other shapes of fuzzy numbers, such as trapezoidal fuzzy numbers, bell-shaped fuzzy numbers, are implemented [21], [24]. The triangular-shape fuzzy number is thus suitable to be applied to our online matching systems for bidding.



Setting the requirement of a buyer
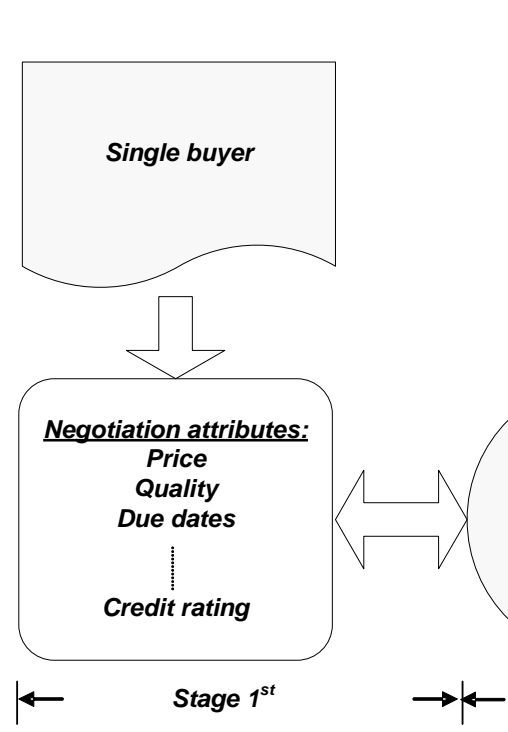

Bidding Process

Process

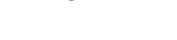

Figure 1: Multi-attribute matching mechanism

\section{Setting bid offers of sellers}

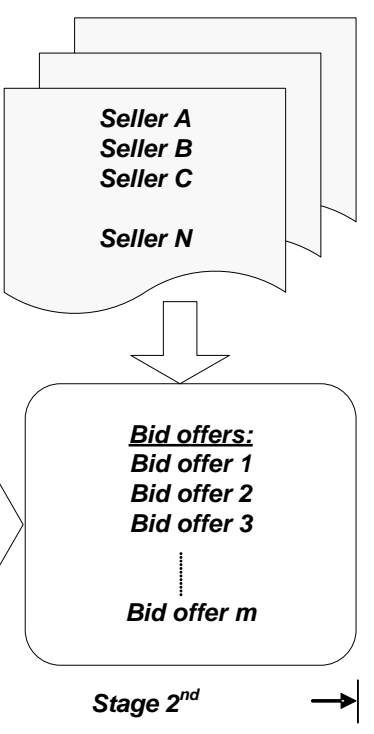

\subsection{The Matching Process}

In a bidding process, the buyer makes a request with the required negotiation attributes, such as price, quality, and due dates, and the respective weights for the attributes. The sellers then respond to the request with their tendered bids, after referring to the terms and weights of attributes requested by the buyer. The matching system then computes and ranks each bid with reference to the negotiation terms and weights. Because the negotiation units such as price, quality, and due dates could be different, the negotiation terms must be normalized to have equivalent size of value before performing the matching operation. After performing the fuzzy arithmetic operations of negotiation terms to obtain triangular fuzzy membership functions, defuzzification is carried out to compute the total 
evaluation value of each seller's bid. The values are sorted as a reference for the buyer to select among the sellers' offers.

Fuzzy negotiation terms of attributes have benefits in the natural representation of human intention; the triangular fuzzy number also makes the mathematical calculation easy to be implemented in a web-site system. The negotiation matching process of the study is described below.

\section{Stage 1 Setting the requirements of a buyer}

The buyer first sets the purchase requirements, such as product name, quantity, specifications, and other negotiation attributes (e.g., price, quality, due dates, and credit rating). In this study, we use triangular fuzzy numbers to represent the fuzzy behavior of negotiation attributes, which have their own specific interval ranges of $X$. In the interval range, an $x \in X$ that is most likely to happen has a membership grade of 1 , while the least likely has 0 . Since the buyer may regard each negotiation attribute differently, he can set diverse weights for the negotiation attributes. Let $n$ represent the number of negotiation attributes and $i \in[1, \cdots \cdots, n]$ the index of negotiation attributes, then the ith negotiation attribute can be represented by a triangular fuzzy number of $A_{i}(x)=\operatorname{tri}\left(x ; a_{i}, b_{i}, c_{i}\right)$, and for $u_{i}, v_{i}, z_{i}$ having values between 0 and 1 , the ith negotiation weight is set as $W_{i}=w\left(u_{i}, v_{i}, z_{i}\right)$. Fuzzy weights can be defined by the buyer with functions like triangles. Those weights can be represented by a set of linguistic fuzzy variables as well. Linguistic variables are the words used in human languages to express the decision maker's feeling of the importance level of attributes with pre-determined triangle values [20]. For example, we can set very important as $w(0.7,0.9,1)$, important as $w(0.5,0.7,0.9)$, fair as $w(0.3,0.5,0.7)$, insignificant as $w(0.1,0.3,0.5)$, etc. In either way, the compound vector forms of the negotiation attributes are then defined as $\overrightarrow{\operatorname{Prj}}\left[A_{1}(x), A_{2}(x), \ldots, A_{n}(x)\right]$ and weights as $\overrightarrow{w P r j}\left[W_{1}, W_{2}, \ldots, W_{n}\right]$.

\section{Stage 2 Setting the bid offers of sellers}

In response to the tendered bid, sellers prepare bid offers according to the product name, quantity and specifications provided by the buyer. The bid offers contain the same kinds of attributes requested by the buyer. Let $m$ be number of bid offers, $j \in[1, \cdots \cdots, m]$ the index of bid offers, $y_{j i}$ the crisp values of the ith negotiation attribute of the $j$ th bid offer. The compound vector form of the $j$ th offer for negotiation attributes of $i$ is then $\overrightarrow{\operatorname{Pro}_{j}}\left[y_{j 1}, y_{j 2}, \ldots, y_{j n}\right]$.

\section{Stage 3 Matching and negotiation}

Negotiation terms have different units, and thus normalization is required before performing the fuzzy negotiation matching process. The normalization fits the value proposed by bid offer $j$ into the fuzzy negotiation term $A_{i}$ of attribute $i$ requested by the seller to reflect the level of fitness of the bid offer to the request. Normalization of $y_{j i}$ into $A_{i}$, denoted by $A_{i}\left(y_{j i}\right)$, has a value between $0 \sim 1$. In this study, $A_{i}$ is a triangular fuzzy number; thus, depending on the value of $y_{j i}, A_{i}\left(y_{j i}\right)$ exists in either of the two forms, as is shown below and Figure 2 (a) and Figure 2 (b):

$$
\begin{aligned}
& A_{i}\left(y_{j i}\right)=\left(y_{j i}-a_{i}\right) /\left(b_{i}-a_{i}\right) \text { for } a_{i} \leq y_{j i} \leq b_{i} \\
& A_{i}\left(y_{j i}\right)=\left(y_{j i}-b_{i}\right) /\left(c_{i}-b_{i}\right) \text { for } b_{i} \leq y_{j i} \leq c_{i}
\end{aligned}
$$

After normalizing the negotiation terms, the normalized offer of $\overline{\operatorname{Pro}_{j}}\left[y_{j 1}, y_{j 2}, \ldots, \mathrm{y}_{j n}\right]$ is then presented as $\overline{n \operatorname{Pro}}\left[A_{1}\left(y_{j 1}\right), A_{2}\left(y_{j 2}\right), \ldots, A_{n}\left(y_{j n}\right)\right]$, which represents the level of fitness of bid offer $j$ from the seller on the respective attributes $i \in[1, \cdots \cdots, n]$ requested by the buyer.

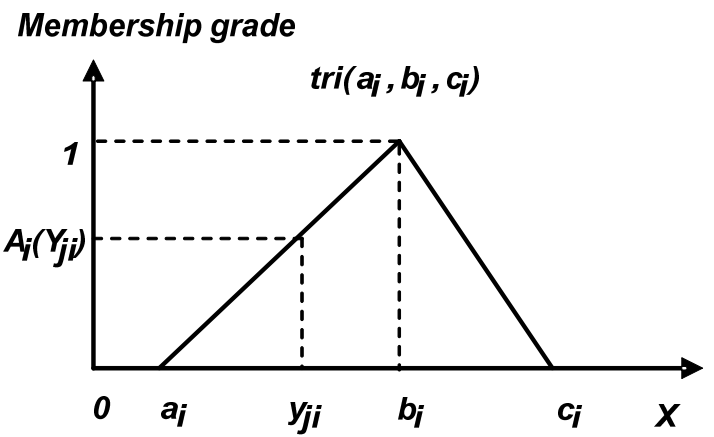

Figure 2 (a): Normalization of condition 1

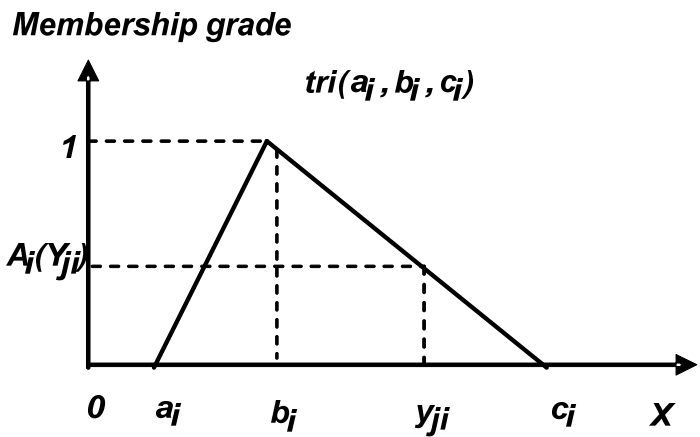

Figure 2 (b): Normalization of condition 2 
After normalization, the values given by the buyer and sellers are calculated to obtain the evaluation value of the sellers' offer. Let $F_{i}\left(\tilde{u}_{i}, \tilde{v}_{i}, \tilde{z}_{i}\right)$ denote the fuzzy number of the jth seller's offer on the ith negotiation attribute and $S_{i}(\tilde{u}, \tilde{v}, \tilde{z})$ the total fuzzy evaluation value of the jth seller's offer. By multiplying $A_{i}\left(y_{j i}\right)$ with the corresponding ith fuzzy negotiation weight $W_{i}=w\left(u_{i}, v_{i}, z_{i}\right)$, the fuzzy evaluation value of the $j$ th offer on the ith negotiation attribute $F_{j}\left(\tilde{u}_{i}, \tilde{v}_{i}, \tilde{z}_{i}\right)$ can be obtained. The total evaluation fuzzy number of bid $j$, denoted as $S_{j}(\tilde{u}, \tilde{v}, \tilde{z})$, can then be determined by adding the fuzzy evaluation values $F_{j}\left(\tilde{u}_{i}, \tilde{v}_{i}, \tilde{z}_{i}\right)$ of all the negotiation attributes for $i \in[1, \cdots \cdots, n]$. Therefore, we have

$$
\begin{aligned}
& F_{j}\left(\tilde{u}_{i}, \tilde{v}_{i}, \tilde{z}_{i}\right)=W \cdot A_{i}\left(Y_{j i}\right) \\
& S_{j}(\tilde{u}, \tilde{v}, \tilde{z})=\sum w\left(u_{i}, v_{i}, z_{i}\right) \cdot A_{i}\left(Y_{j i}\right)=\sum F_{j}\left(\tilde{u}_{i}, \tilde{v}_{i}, \tilde{z}_{i}\right) .
\end{aligned}
$$

Through the defuzzification such as Eq. (2), the total evaluation value of the jth bid offer $S_{j}(\tilde{u}, \tilde{v}, \tilde{z})$, denoted as $d f\left(S_{j}(\tilde{u}, \tilde{v}, \tilde{z})\right)$, can be obtained. Specifically, larger total evaluation value of a bid offer means the bid offer fits more closely to the tendered bid. Therefore, the defuzzified value of bid offers $j \in[1, \cdots \cdots, m]$ are computed and ranked to obtain the largest total evaluation value as $\max _{j \in[1, \ldots, m]}\left[d f\left(S_{1}(\tilde{u}, \tilde{v}, \tilde{z})\right), \ldots, d f\left(S_{m}(\tilde{u}, \tilde{v}, \tilde{z})\right)\right]$.

The tendered bid with the highest total evaluation value is recommended by the matching system to the buyer. If the buyer is satisfied with the recommended bid offer, the negotiation process is terminated; otherwise, an adjustment in the bid offers from the sellers is required, and the negotiation process enters into the aftermath negotiations.

\subsection{Conducting Aftermath Negotiations}

During the negotiation process, the seller and the buyer have their own opinions, so a good match usually requires several times of negotiations. In addition, when the negotiation has reached an impasse, the seller and the buyer cannot conclude with the transaction. To restart the negotiation mechanism when the impasse happens, this study utilizes the aftermath negotiations, where the buyer can set a target total evaluation value to re-calculate a negotiation value specified by the buyer when the buyer is dissatisfied with the negotiation term (e.g., price is high or quality is poor, etc.) proposed by the seller who wins the tendered bid. Based on the re-calculated value of the negotiation term, the buyer and the seller can negotiate again to reach a matched offer. In the aftermath negotiations, the buyer initiates and sellers respond.

Assume the buyer wishes to renegotiate with the seller on the attribute $i$ of bid offer $j$. Then, the renegotiated bid offer has the same vector form as the original offer except for the attribute $i$ which is set to have a normalized value of $\delta$. By multiplying the renegotiated bid offer with the weight vector and set the resultant defuzzification value equal to the expected value of the buyer, we can obtain the value of $\delta$. With the inverse function in Eq.(3) or (4), the should-be value $\pi$ for the attribute $i$ can be obtained.

The above procedure is straightforward and contains no computation difficulty as applied to general web-sites. In many a case, the aftermath negotiation may take more than one round when the seller disagrees with the recalculated value. In that situation, the negotiation may continue to work on other attributes or may even resort to other sellers. Most previous studies regarding negotiations (e.g., [13]-[14], [29]) are to conclude a final equilibrium condition satisfied by both parties. Seldom research has been studied on aftermath negotiations until recently when Leskela et al. [26] proposed a quantity decision support (QDS) algorithm to resolve the problem of finding no solution after solving the winner determination problem (WDP) in a combinatorial reverse auction environment. However, their algorithms are quite tedious and require solving at least two WDP models, which is an NP-complete problem [26], in a single round of negotiation. In contract, our aftermath negotiations are much simpler when encountering similar situations in reverse auctions.

\subsection{Orientations of Negotiation Attributes}

The negotiation between the buyer and seller occurs when their decision-making orientations are the opposite. The negotiation orientation can be divided into two scenarios. In the first scenario, a smaller value of the negotiation attribute meets the expectation of the buyer higher, while the seller expects more for the higher value of the negotiation attribute. For example, the buyer hopes that the price is lower, while the seller does the opposite. For lower prices, the buyer perceives higher utility, and thus larger membership values, and vice versa. The seller has a 
membership function for price contrary to that of the buyer, where higher prices induce higher utility and larger membership values. In the second scenario, higher value of the negotiation attribute meets the expectancy of the buyer more, while the seller expects more for the lower value of the negotiation attribute. The attributes of quality is one of the examples. If the orientation of negotiation attributes for the seller and buyer is the same, they will choose a value that mutually meets the highest expectation of each other without negotiation, and the membership grade of the attributes has a value of 1 .

The above discussion reveals that the negotiation attributes included in the negotiation mechanism are the ones that have opposite orientation for the buyer and seller, such as price, quality (e.g., factory output quality), order due dates, credit rating (e.g., financial capacity, past transaction credit, etc.). Figure 3 shows the possible triangular fuzzy numbers of the four negotiation attributes from the views of the buyer and the seller. This study lays emphasis on the discussion and construction of the negotiation mechanism, so straightforward convention is introduced for setting the attribute values. For ease of computation and setting, the quantitative value of the quality and credit rating is set between 0 and 10 .

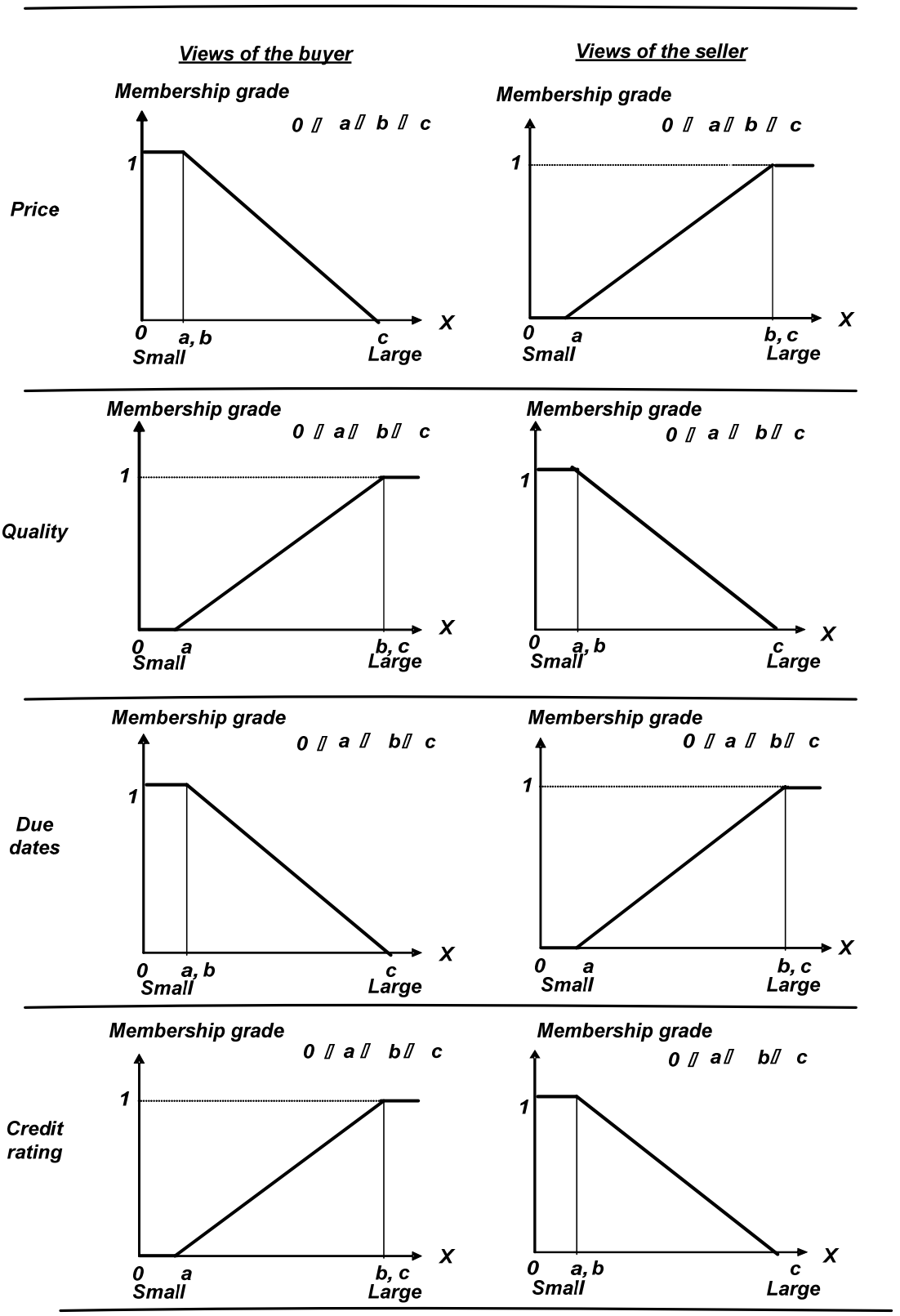

Figure 3: Preference orientation of the buyer and the seller 


\section{Applying the Fuzzy Matching and Negotiation Mechanism}

To demonstrate the implementation of the mechanism, in this section, we will present a negotiation matching example by firstly demonstrating the matching mechanism with numbers and then showing the aftermath negotiation procedures and lastly presenting a prototype of an online fuzzy negotiation system (FNS) for validation.

\subsection{A Demonstration of the Matching Mechanism}

We follows the three stages of the matching processes to demonstrate the implementation of the mechanism, where the requirement values of the buyer and the terms of bid offers from sellers are assumed given.

Stage 1 Setting of the requirements of the buyer Assume the buyer requests the range of the product price (negotiation attribute $i=1$ ) is $\$ 500 \sim 750$, the quality level $(i=2) 3 \sim 7$, the due date $(i=3)$ 5 12 days, and the credit rating (i=4) 5 9. Then the request for the buyer becomes

$$
\overrightarrow{\operatorname{Prj}}[\operatorname{tri}(x ; 500,500,750), \operatorname{tri}(x ; 3,7,7), \operatorname{tri}(x ; 5,5,12), \operatorname{tri}(x ; 5,9,9)]
$$

Different negotiation attributes for the buyer are weighted diversely. As was mentioned in section 2, the importance levels of negotiation attributes perceived by the buyer are modeled as weights. Assume the buyer sets triangular fuzzy numbers for the price as $W_{1}=w(0.5,0.8,0.9)$, quality $W_{2}=w(0.4,0.6,0.7)$, due date $W_{3}=w(0.3,0.4,0.5)$ and credit rating $W_{4}=w(0.6,0.7,0.9)$. Then fuzzy attribute weights are displayed as:

$$
\overrightarrow{w P r j}[w(0.5,0.8,0.9), w(0.4,0.6,0.7), w(0.3,0.4,0.5), w(0.6,0.7,0.9)]
$$

Stage 2 Setting the bid offers of sellers

In this section of the example, we assume that there are three bid offers from the sellers. The first bid offer is proposed by SellerA (offer $j=1$ ), in which the product price is NT $\$ 600$, quality level 5 , due date 7 days, and credit rating 6 . The bid offer of SellerA becomes $\overrightarrow{\operatorname{Pro}}_{1}[600,5,7,6]$. Similarly, we assume that the bid offer from SellerB (offer $j=2$ ) is $\overline{\operatorname{PrO}_{2}}[800,9,10,8]$ and from Seller $C($ offer $j=3)$ is $\overrightarrow{\operatorname{Pro}_{3}}[700,6,12,9]$.

\section{Stage3 Matching and negotiation}

With Eq.(3) or (4), the negotiation terms of the bid offers from the sellers can be normalized. Take SellerA's bid offer as an example. Based on the buyer's requirement as described in Eq.(7), the normalized negotiation terms of SellerA becomes $\overrightarrow{n \operatorname{Pro}_{1}}[0.6,0.5,0.714,0.25]$, which are displayed in Figure 4 with the associated fuzzy weights shown in Eq.(8). Bid offer 2 from SellerB is then $\overline{n \operatorname{PrO}_{2}}[0,1,0.286,0.75]$ and bid offer 3 from SellerC is $\overrightarrow{\mathrm{PrO}_{3}}[0.2,0.75,0,1]$.

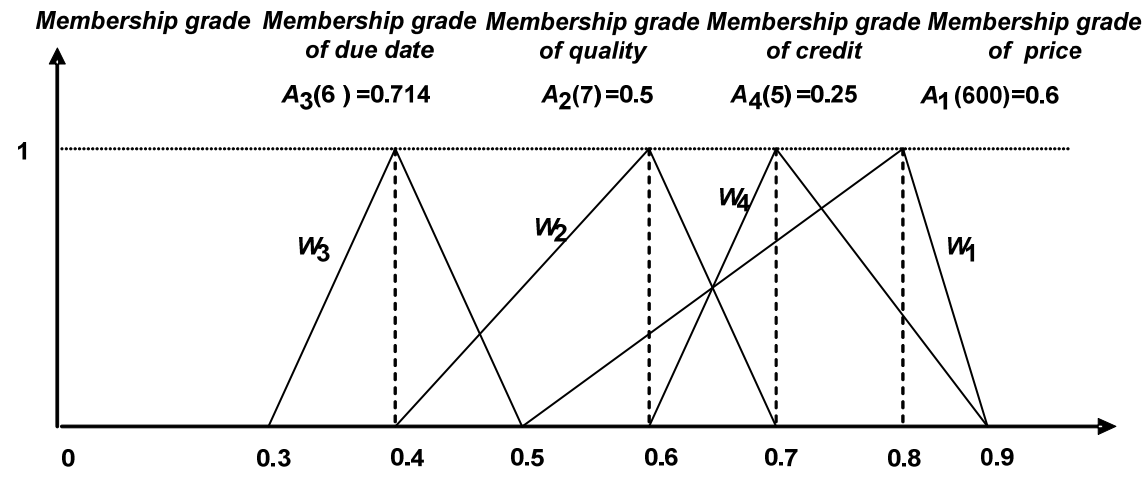

Figure 4: Normalized terms and fuzzy weights

The fuzzy evaluation function of bid offer $j$ on negotiation attribute $i$, denoted as $F_{i}\left(\tilde{u}_{i}, \tilde{v}_{i}, \tilde{z}_{i}\right)$, can be computed by Eq.(5); thus the four fuzzy evaluation functions of SellerA are $F_{1}\left(\tilde{u}_{1}, \tilde{v}_{1}, \tilde{z}_{1}\right)=W_{1} \cdot A_{1}\left(Y_{11}\right)=F_{1}(0.3,0.48,0.54)$, and $F_{1}\left(\tilde{u}_{2}, \tilde{v}_{2}, \tilde{z}_{2}\right)=F_{1}(0.2,0.3,0.35), F_{1}\left(\tilde{u}_{3}, \tilde{v}_{3}, \tilde{z}_{3}\right)=F_{1}(0.21,0.28,0.35)$, and $F_{1}\left(\tilde{u}_{4}, \tilde{v}_{4}, \tilde{z}_{4}\right)=F_{1}(0.15,0.175,0.225)$. The 
fuzzy evaluation function $F_{i}\left(\tilde{u}_{i}, \tilde{v}_{i}, \tilde{z}_{i}\right)$ is a triangular fuzzy number, thus the sum of evaluation functions is formed by adding up the corresponding apexes of the triangles. The total fuzzy evaluation value of function $S_{1}$ of bid offer 1 by SellerA can be determined by Eq.(6) as $S_{1}(\tilde{u}, \tilde{v}, \tilde{z})=\sum_{i=1}^{4} F_{1}\left(\tilde{u}_{i}, \tilde{v}_{i}, \tilde{z}_{i}\right)=F_{1}(0.86,1.235,1.465)$, and is depicted in Figure 5. The bid offers from SellerB and SellerC can also be obtained as $S_{2}(\tilde{u}, \tilde{v}, \tilde{z})=F_{2}(0.94,1.24,1.52)$ and $S_{3}(\tilde{u}, \tilde{v}, \tilde{z})=F_{3}(1,1.31,1.61)$.



Figure 5: Total fuzzy evaluation value of bid offer 1

Table 1: Ranking of sellers' bid offers

\begin{tabular}{|l|c|l|c|c|}
\hline $\begin{array}{c}\text { Buyer } \boldsymbol{I} \\
\text { Sellers }\end{array}$ & \multicolumn{1}{|c|}{$\begin{array}{c}\text { Bid offers } \\
{[(\text { price) (quality) (due date) (credit rating)] }}\end{array}$} & \multicolumn{1}{c|}{ Normalization } & $\begin{array}{c}\text { Total } \\
\text { evaluation value }\end{array}$ & Ranking \\
\hline Buyer & {$[(500 \sim 750)(3 \sim 7)(5 \sim 12)(5 \sim 9)]$} & {$[(1,0)(0,1)(1,0)(0,1)]$} & - & - \\
\hline SellerA & {$[(600)(5)(7)(6)]$} & {$[(3 / 5)(1 / 2)(5 / 7)(1 / 4)]$} & 1.19 & 3 \\
\hline SellerB & {$[(800)(9)(10)(8)]$} & {$[(0)(1)(2 / 7)(3 / 4)]$} & 1.23 & 2 \\
\hline SellerC & {$[(700)(6)(12)(9)]$} & {$[(1 / 5)(3 / 4)(0)(1)]$} & 1.31 & 1 \\
\hline
\end{tabular}

\subsection{Conducting Aftermath Negotiations}

After obtaining the results shown in Table 1, if the buyer has the lowest threshold value for the total evaluation value, say 1.5, then none of the bid offer meets the requirement. After observation, if the buyer thinks the price of the bid offer by SellerC can be lower to achieve the threshold value, the buyer, with this aftermath negotiation function, can ask the seller to adjust the price without changing other attribute values and still have a total evaluation value of 1.5 or above.

For SellerC, the renegotiated bid offer is $j=\operatorname{adj}_{3}$ (here bid offer of SellerC is 3). The normalized bid offer in vector form is thus expressed by $\overline{n P r o}{ }_{a_{j} j_{3}}[\delta, 0.75,0,1]$. Substitute the weights shown in Eq.(8) and the above normalized bid offer into Eq.(6), we have $S_{3}(\tilde{u}, \tilde{v}, \tilde{z})=S_{3}(0.5 \delta+0.9,0.8 \delta+1.15,0.9 \delta+1.425)$. Since $d f\left(S_{\text {adj }_{3}}(\tilde{u}, \tilde{v}, \tilde{z})\right)$ is set as 1.5 , from Eq. (2), $\delta=0.466$. Substituting $\delta$ into the inverse function in Eq.(3) or (4), the should-be value for price $\pi$ can be obtained as $\pi=\$ 633.59$.

From the above demonstration regarding the matching computation, when the total evaluation value of the bid offer of SellerC is adjusted to as 1.5 and other terms of attributes remain unchanged, the original price is required to be lowered from $\$ 700$ to $\$ 633.59$, which is the highest price the buyer would negotiate with SellerC. If SellerC accepts this proposition, the matching between the buyer and seller is reached; otherwise, the buyer can continue adjusting and negotiating or giving up the bid offer of SellerC. Of course, the buyer can negotiate with other sellers as well or make negotiations on other attributes, such as quality, due date or credit rating.

\subsection{A Fuzzy Negotiation System Demonstration}

After presenting the development and demonstration processes of the mechanism, we constructed an online FNS as a prototype of the mechanism for reverse auctions based upon the three negotiation matching stages proposed in this study. Developing a prototype system with simulation data is regarded as a useful evaluation process for verifying the effectiveness and efficiency of the mechanism built for system development [19], [28], [37]. In 
constructing the FNS, it was observed that fuzzy computation of triangular fuzzy numbers is straightforward, so it is very applicable to include fuzziness in the design of an online bidding website. The FNS website was constructed on the Microsoft Windows 2000 Server or after, and applied the Active Server Page (ASP) programming language with Microsoft Access and SQL syntax. Interested readers can access the FNS web site (Site 1).

Since the computation of the proposed mechanism is simple, the whole matching and negotiation processes can be easily applied in the website design through SQL syntax. After the FNS was constructed, we performed reverse auctions with simulation data for verification [19], [37]. Figure 6 shows the matching results of the bid offers, where the FNS calculates the total evaluation values of the sellers' offers on the buyer's bid No.134. In the figure, bid offer $j=8$ proposed by Blue Ocean Corp. has a normalized vector form of $\overline{n \operatorname{Pro}_{8}}[0.83,0.33,0.75,1]$, and it has the largest total evaluation value among all the sellers' bids of offer as 2.53. If the buyer is happy with this winning bid or if the bidding conditions of the matching system stipulate that a bid concludes automatically with the highest bid offer, the bid is finalized as it is; otherwise, the buyer can continue to the aftermath negotiations.

In the aftermath negotiations, the buyer can re-negotiate with Victor Corp. of $j=5$ based on the long-term cooperation relationship with the company. By setting the total evaluation value of bid offer $j=5$ from 2.30 to 2.53 or bigger, and selecting the "price" as the negotiation attribute, the system will come out with the adjusted price of 812,423 . This means that if the price is reduced from 820,000 to 812,423 for the bid offer $j=5$, the total evaluation value becomes 2.53 when the values of other attributes remain unchanged. The buyer can then negotiate with Victor Corp. on bid offer $j=5$. Of course, the above aftermath negotiation method is applicable to the adjustment of the other bid offers and/or negotiation attributes of the seller.

The evaluation of our proposed mechansim demonstrates its effectiveness in performing multi-attribute matching and negotiation for sealed-bid reverse actions [28], [37]. The construction of the FNS also shows the efficiency of our mechanism in applying fuzzy theories in expressing the vagueness of human judgment and preferences as attributes for the matching of bid offers [28], [37].

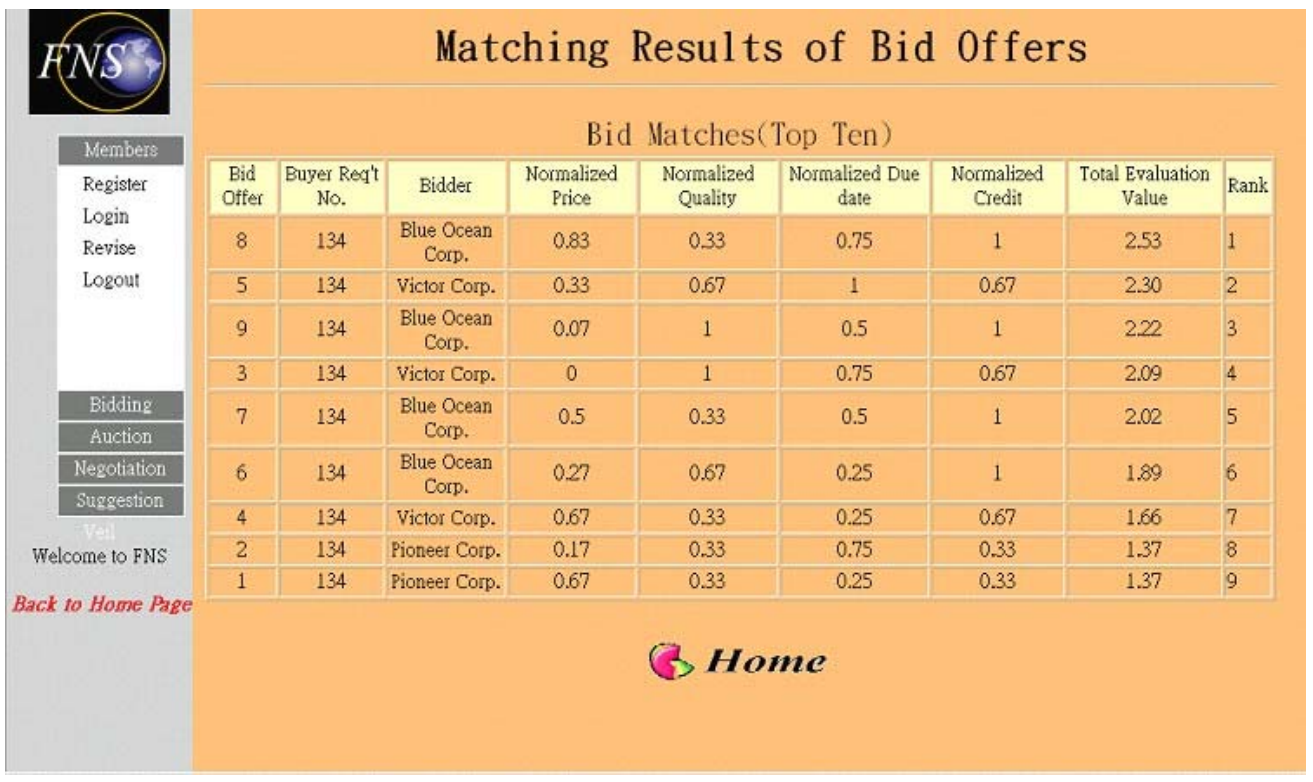

Figure 6: FNS matching results of bid offers

\section{Conclusions and Suggestions}

The extensive application of electronic commerce enables enterprises to reduce the transaction cost via Internetbased trading activities and to improve the operational efficiency (e.g., [1], [3]-[4], [16], [23]). However, the transactions between enterprises are complicated. The seller and buyer need to evaluate and negotiate with each other and consider many factors during the transaction, so as to conclude a mutually beneficial agreement. The rapid development of online reverse auction is attributed to the price-saving effect experienced by the buyer and seller and the improvement in the transaction processes. However, previous research also shows that the online reverse auction may result in negative evaluations, and may affect the long-term cooperation relationship between the seller and buyer [8]-[9], [16], [22]. Daly and Nath [8]-[9] suggested that online reverse auction systems could be designed to meet the needs of the long-term investment and business relationship between enterprise partners, such as the subsidization for the cooperative partnership or the bidding loss, or even providing the mechanism for re-negotiating the final bid prices and specifications. 
In industry application, direct input goods and outsourcing of engineering projects account for the major amount of business purchasing costs [6], [10], [33]. However, previous research was mostly conducted in an open-cry and multiple rounds of auctions, which is applicable to indirect input goods like MRO types of goods and is not appropriate for direct input goods [2], [5]-[6], [10]. The auctioneer and the bidders for indirect input goods are selfinterested and the negotiation process usually involves multiple rounds. To reach an agreement in a reasonable time, both parties may set up a negotiation protocol to deal with this competitive situation with a discount factor for one additional round of negotiation [13]-[14], [29]. Moreover, in some cases, the negotiation process may not conclude with any results [13], [29].This study develops a fuzzy multi-attribute mechanism that the matching of sealed-bids is completed in a single round. In addition to the price attribute, the negotiation attributes of quality, due dates, and credit rating, which may affect the matching, are considered as well. The total evaluation values of the bid offers are found through the fuzzy matching mechanism with simple arithmetic operations which can be easily embedded in a reverse auction website. Since this mechanism is suitable for direct input goods, both the auctioneer and the bidders work in a cooperative manner (of course, among bidders, it is still in a competitive manner) with a hope to maintain the long-term relationship that is not easily built [8]-[9], [25]. Besides the attributes considered in the reverse auction, time accuracy and delivery assurance resulted from the process of reverse auctions are also major concerns for direct input goods because they affect the operations of production lines directly. Our aftermath negotiation is established to allow the buyer to flexibly negotiate with sellers on bid offers to speed up the reverse auction process and to assure arriving at a conclusion. In addition, the aftermath negotiation is conducted on the basis of cooperation and trust between the two parties to create a win-win situation, which differentiates our research from those studies based on self-interested negotiation parties.

Practitioners can easily construct a sealed-bid reverse auction system for procuring their direct input goods in a fuzzy setting following the matching process and the aftermath negotiations exhibited in section 3.1 and 3.2. The FNS shown in section 4.3 is a prototype system for this purpose and is developed with fundamental software that is available handily. By developing the FNS, our proposed mechanism is evaluated through building a prototype system and through performing some simulation studies for reverse auctions [19], [28], [37]. For the existing procurement system of a company, either the buy-side or the neutral e-marketplace system, our model can also be readily appended to since the coding required for our mechanism is straightforward without resorting to specialty software for complex computation.

The mechanism developed in this study utilizes negotiation attributes to arrive at an evaluation value for bid ranking, and those negotiation attributes represent the specifications of bids and should be transformed numerically before being implemented in the mechanism. For standardized products of direct input goods, specifications can be easily transformed into numerals. However, for non-standardized products, specifications are variant and usually cannot be handily converted into numerals in an allowable time during the procurement process, and this limits their application to our mechanism.

Even this fuzzy negotiation mechanism is derived in the B2B online reverse auctions, it can be easily converted to be applied in B2C and C2C matching processes. For example, this mechanism can be used in B2C matching operations such as the house rental or real estate services, the car rental or sale services, the travel scheduling, etc.; it can be used in $\mathrm{C} 2 \mathrm{C}$ matching operations like personal stuffs auctions or bidding. In addition, if the roles of the buyer and seller are interchanged such that a single seller is the bid setter, and numerous buyers offer the bids, then this reverse auction system becomes an auction system.

\section{Websites List}

Site 1: Fuzzy Negotiation System

http://140.130.128.90/ec/fns

\section{References}

[1] U. Arnold, H. Karner, and M. Schnabel, Target-oriented use of strategic sourcing tools: A critical analysis crating process awareness for electronic reverse auctions, Journal of Purchasing and Supply Management, vol. 11, no. 2-3, pp. 116-128, 2005

[2] M. Bichler and J. Kalagnanam, Configurable offers and winner determination in multi-attribute auctions, European Journal of Operational Research, vol. 160, no. 2, pp. 380-394, 2005.

[3] J. Carbone, Debate rages over use of e-auction for components, Purchasing, vol. 132, no. 19, pp. 48-52, 2003.

[4] C. R. Carter, L. Kaufmann, S. Beall, P. L. Carter, T. E. Hendrick, and K. J. Petersen, Reverse auctions grounded theory from the buyer and supplier perspective, Transportation Research Part E: Logistics and Transportation Review, vol. 40, no. 3, pp. 229-254, 2004.

[5] S. C. Chang, Reverse electronic auctions: Cases in Taiwan's high-tech industry, Technology in Society, vol. 29, no. 4, pp. 490-496, 2007.

[6] C. B. Cheng, Solving a sealed-bid reverse auction problem by multiple-criterion decision-making methods, Computers and Mathematics with Applications, vol. 56, no. 12, pp. 3261-3274, 2008. 
[7] C. B. Cheng, C. H. Chan, and K. C. Lin, Intelligent agents for e-marketplace: Negotiation with issue trade-offs by fuzzy inference systems, Decision Support Systems, vol. 42, no. 2, pp. 626-638, 2006.

[8] S. P. Daly and P. Nath, Reverse auctions for relationship marketers, Industrial Marketing Management, vol. 34, no. 2, pp. 157-166, 2005 .

[9] S. P. Daly and P. Nath, Reverse auctions and buyer - seller relationships: A rejoinder to Emiliani and Stec's commentary, Industrial Marketing Management, vol. 34, no. 2, pp. 173-176, 2005.

[10] E. David, R. Azoulay-Schwartz, and S. Kraus, Bidding in sealed-bid and English multi-attribute auctions, Decision Support Systems, vol. 42, no. 2, pp. 527-556, 2006.

[11] V. Ervasti and R.-L. Leskela, Allocative efficiency in simulated multiple-unit combinatorial auctions with quantity support, European Journal of Operational Research, vol. 203, no. 1, pp. 251-260, 2010.

[12] M. L. Emiliani, Regulating B2B online reverse auctions through voluntary codes of conduct, Industrial Marketing Management, vol. 34, no. 5, pp. 526-534, 2005.

[13] S. S. Fatima, M. Wooldridge, and N. R. Jennings, A comparative study of game theoretic and evolutionary models of bargaining for software agents, Artificial Intelligence Review, vol. 23, no. 2, pp. 185-203, 2005.

[14] S. S. Fatima, M. Wooldridge, and N. R. Jennings, Multi-issue negotiation with deadlines, Journal of Artificial Intelligence Research, vol. 27, no. 1, pp. 381-417, 2006

[15] A. Foroughi, M. Kocakulah, and J. Williams, A framework for electronic reverse auction (eRA) research, Journal of Internet Commerce, vol. 6, no. 3, pp. 45-74, 2007.

[16] T. F. Gattiker, X. Huang, and J. L. Schwarz, Negotiation, email, and Internet reverse auctions: How sourcing mechanisms deployed by buyers affect suppliers' trust, Journal of Operations Management, vol. 25, no. 1, pp. 184-202, 2007.

[17] W. Grey, T. Olavson, and D. Shi, The role of e-marketplaces in relationship-based supply chains: A survey, IBM Systems Journal, vol. 44, no. 1, pp. 109-123, 2005.

[18] X. Gu and Q. Zhu, Fuzzy multi-attribute decision-making method based on eigenvector of fuzzy attribute evaluation space, Decision Support Systems, vol. 41, no. 2, pp. 400-410, 2006.

[19] A. R. Hevner, S. T. March, and J. Park, Design science in information systems research, MIS Quarterly, vol. 28, no. 1, pp. 75-105, 2004.

[20] C. L. Hwang and K. Yoon, Multiple Attribute Decision Making, Methods and Applications - A State-of-the-Art Survey. New York, NY: Springer-Verlag, 1981.

[21] J. S. Jang, C. T. Sun, and E. Mizutani, Neuro-Fuzzy and Soft Computing: A Computational Approach to Learning and Machine Intelligence. Upper Saddle River, NJ: Prentice-Hall, 2004.

[22] S. D. Jap, Online eRAs: Issues, themes, and prospects for the future, Journal of the Academy of Marketing Science, vol. 30, no. 4, pp. 45-74, 2002.

[23] M. Jin and S. D. Wu, Supplier coalitions in on-line reverse auction: Validity requirements and profit distribution scheme, International Journal of Production Economics, vol. 100, no. 2, pp. 183-194, 2006.

[24] J. G. Klir and B. Yuan, Fuzzy Sets and Fuzzy Logic: Theory and Applications. Upper Saddle River, NJ: Prentice Hall, 1995.

[25] N. Kumar, L. Scheer, and J. B. Steenkamp, The effects of supplier fairness on vulnerable resellers, Journal of Marketing Research, vol. 32, no. 1, pp. 54-65, 1995.

[26] R.-L. Leskela, J. Teich, H. Wallenius, and J. Wallenius, Decision support for multi-unit combinatorial bundle auctions, Decision Support Systems, vol. 43, no. 2, pp. 420-434, 2007.

[27] F. Nassiri-Mofakham, M. A. Nematbakhsh, N. Ghasem-Aghaee, and A. Baraani-Dastjerdi, A heuristic personality-based bilateral multi-issue bargaining model in electronic commerce, International Journal of Human-Computer Studies, vol. 67, no. 1, pp. 1-35, 2009.

[28] K. Peffers, T. Tuunanen, M. A. Rothenberger, and S. Chatterjee, A design science research methodology for information systems research, Journal of Management Information Systems, vol. 24, no. 3, pp. 45-77, 2007

[29] S. D. Ramchurn, C. Sierra, L. Godo, and N. R. Jennings, Negotiating using rewards, Artificial Intelligence, vol. 171, no. 10-15, pp. 805-837, 2007.

[30] D.-H. Shih, S.-Y. Huang, and D. C. Yen, A new reverse auction agent system for m-commerce using mobile agents, Computer Standards and Interfaces, vol. 27, no. 4, pp. 383-395, 2005.

[31] A. Smart and A. Harrison, Reverse auctions as a support mechanism in flexible supply chains, International Journal of Logistics, vol. 5, no. 3, pp. 275-284, 2002.

[32] J. E. Teich, H. Wallenius, J. Wallenius, and O. R. Koppius, Emerging multiple issue e-auctions, European Journal of Operational Research, vol. 159, no. 1, pp. 1-16, 2004.

[33] J. E. Teich, H. Wallenius, J. Wallenius, and A. Zaitsev, A multi-attribute e-auction mechanism for procurement: theoretical foundations, European Journal of Operational Research, vol. 175, no. 1, pp. 90-100, 2006

[34] F. Teuteberg, Experimental evaluation of a model for multilateral negotiation with fuzzy preferences on an agent-based marketplace, Electronic Markets, vol. 13, no. 1, pp. 21-32, 2003.

[35] R. Wang, Bidding and renegotiation in procurement auctions, European Economic Review, vol. 44, no. 8, pp. 1577-159, 2007.

[36] Y. Wang, K. L. Tan, and J. Ren, PumaMart: A parallel and autonomous agents based Internet marketplace, Electronic Commerce Research and Applications, vol. 3, no. 3, pp. 294-310, 2004.

[37] J.-H Wu, A design methodology for form-based knowledge reuse and representation, Information \& Management, vol. 46, no. 7, pp. 365-375, 2009. 